Slavica

bruxellensia

\section{Slavica bruxellensia}

Revue polyphonique de littérature, culture et histoire

slaves

$8 \mid 2012$

Migration(s) et Exil(s)

\title{
Publier en exil : écrivains et imprimeurs polonais à Bruxelles, 1830-1870
}

\section{Idesbald Goddeeris}

\section{OpenEdition}

\section{Journals}

Édition électronique

URL : http://journals.openedition.org/slavica/1185

DOI : 10.4000/slavica. 1185

ISSN : 2034-6395

\section{Éditeur}

Université libre de Bruxelles - ULB

\section{Référence électronique}

Idesbald Goddeeris, "Publier en exil : écrivains et imprimeurs polonais à Bruxelles, 1830-1870 »,

Slavica bruxellensia [En ligne], 8 | 2012, mis en ligne le 15 juin 2012, consulté le 22 septembre 2020.

URL : http://journals.openedition.org/slavica/1185; DOI : https://doi.org/10.4000/slavica.1185

Ce document a été généré automatiquement le 22 septembre 2020.

\section{(c) $(1) \&$}

Les contenus de Slavica bruxellensia sont mis à disposition selon les termes de la Licence Creative Commons Attribution - Pas d'Utilisation Commerciale - Pas de Modification 3.0 France. 


\title{
Publier en exil : écrivains et imprimeurs polonais à Bruxelles, 1830-1870
}

\author{
Idesbald Goddeeris
}

1 La Belgique du XIX ${ }^{e}$ siècle a l'image d'un pays très hospitalier. A la veille du XXI ${ }^{e}$ siècle, des historiens se rallient encore à cette réputation déjà développée et répandue au XIX ${ }^{e}$ et $\mathrm{XX}^{\mathrm{e}}$ siècles ${ }^{1}$. En 2000, Claire Billen l'a propagée de la manière suivante :

De par la franchise et l'ouverture extrêmes que sous-tendaient le texte de la constitution, il semblait bien que le jeune état belge fût un paradis pour quiconque avait été expulsé de son pays pour cause

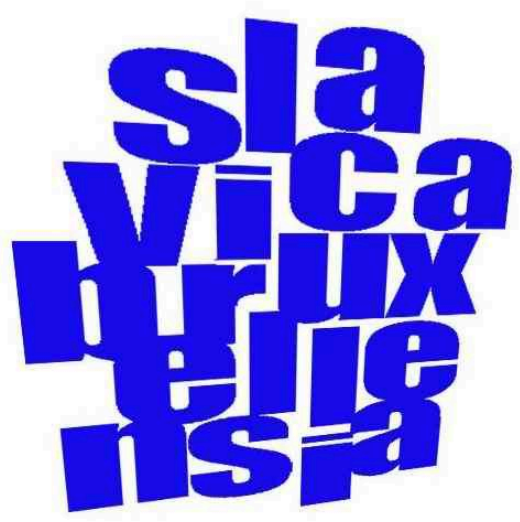
de convictions trop radicales ou non conformistes, ou pour quiconque ressentait un besoin de réflexion politique et dès lors désirait prendre ses distances par rapport à la trop dure réalité. L'attrait de la Belgique augmenta peut-être encore lorsqu'en 1833, un article de loi rendit impossible l'extradition pour cause de délit politique. ${ }^{2}$

Néanmoins, cette hospitalité belge a été depuis mise doute. Ce sujet a fait l'objet d'importants travaux de recherche. En 1978, Jean Stengers écrivait qu'on «ne saurait parler de la Belgique du XIX siècle sans évoquer sa réputation, pour les étrangers, de "terre d'accueil" : les réfugiés politiques, les exilés y ont bénéficié d'un régime qui, pour l'Europe du temps, était particulièrement libéral $»^{3}$. Mais lors d'un congrès international en 1991, sa disciple Anne Morelli a brisé cette image en la qualifiant de " toute relative, sinon mythologique ${ }^{4}$.

2 Un élément spécifique de cette hospitalité n'a pas encore été étudié de manière approfondie. La Belgique jouissait également d'une réputation de centre d'édition. Des 
exilés de divers pays imprimaient leurs pamphlets et leurs livres en Belgique. Les communistes allemands ${ }^{5}$ et les antibonapartistes français ${ }^{6}$ sont les plus connus ${ }^{7}$, mais les Russes également publièrent intensément en Belgique. Les adversaires du régime du tsar éditèrent, au début des années 1860, des périodiques comme Le Véridique, Listok et Svobodnoe Slovo. Le dissident Aleksandr I. Herzen envisagea à plusieurs reprises de déménager le siège de son journal populaire Kolokol de Londres à Bruxelles. Pour leur part, les autorités officielles russes à Bruxelles éditaient leur revue intitulée Le Nord ${ }^{8}$.

3 Sur le plan de l'édition et de la publication, la réputation d'accueil de la Belgique ne semble donc pas être un mythe, comme l'affirme Morelli. Comment doit-on comprendre la position de la Belgique, c'est ce que cet article examinera. La situation des exilés polonais en Belgique dans les années 1830-1870 sera la perspective adoptée dans cet examen.

$4 \quad \mathrm{Au} \mathrm{XIX}^{\mathrm{e}}$ siècle, les Polonais se sont à plusieurs reprises révoltés contre les divisions de leur pays par l'Autriche, la Prusse et la Russie. Une première grande insurrection nationale éclata à Varsovie le 29 novembre 1830. Elle avait été inspirée par deux autres révoltes en Europe : les " Trois Glorieuses » à Paris, qui avaient renversé les Bourbons réinstallés en 1815 sur le trône de France, et la Révolution belge d'août et septembre 1830 contre le roi hollandais Guillaume $\mathrm{I}^{\mathrm{er}}$. Les Polonais furent moins chanceux que les Français et les Belges. Après un peu plus de neuf mois de combat, Varsovie tomba finalement le 8 septembre 1831. Environ sept mille officiers, politiciens et intellectuels émigrèrent vers l'ouest afin de continuer leur lutte en exil. Cette migration fut appelée Wielka Emigracja (Grande Emigration) et comprenait la crème des personnalités culturelles et politiques de la Pologne du XIXe siècle, comme Frédéric Chopin, Adam Mickiewicz, Adam Jerzy Czartoryski et Joachim Lelewel. La plupart des émigrés se dirigèrent vers la France, mais une partie choisit assez rapidement d'autres pays, dont la Belgique, où vécurent quelques centaines d'entre eux'.

5 Par la suite, dans une tradition toute romantique, quelques nouvelles révoltes éclatèrent en Pologne, notamment en 1846 à Cracovie (en Autriche). Cette révolte fut rapidement réprimée mais deux ans plus tard les Polonais participaient déja au Printemps des peuples, à Poznań (en Prusse), en Galicie (la Pologne autrichienne), mais également en Sicile et à Florence, à Berlin et à Francfort. Lorsque les révoltes furent vaincues, les insurgés polonais se dirigèrent à nouveau vers l'ouest. Une nouvelle grande vague d'émigration politique eut lieu après l'Insurrection de janvier (1863-64), la dernière grande révolte polonaise contre le tsar. Cette émigration d'après janvier amena un grand nombre de nouveaux proscrits vers l'ouest ${ }^{10}$.

6 Cette article se propose d'analyser la Belgique comme centre d'édition pour les exilés polonais dans les années 1830-1870. Nous le ferons en deux étapes. Dans un premier temps, nous examinerons de plus près les imprimeries polonaises à Bruxelles en nous posant les questions suivantes: Combien de Polonais ont édité et imprimé des livres en Belgique? qui parmi eux parvint à monter une affaire solide qui réussit? Quels furent les facteurs qui contribuèrent à leur succès? Les publications polonaises furent-elles éditées en Belgique ou les écrivains polonais qui se trouvaient dans ce pays durent-ils collaborer avec des imprimeurs belges? Avaient-ils une réputation internationale et donnaient-ils du travail aux Polonais qui n'étaient pas sur le territoire belge? La réponse à ces différentes questions nous donnera un premier aperçu de la Belgique comme pays d'éditeurs. 
7 Dans un second temps, nous nous focaliserons sur les auteurs et leurs publications. Sur base de données bibliographiques, nous observerons quels livres polonais furent publiés en Belgique par des auteurs polonais. Pour ce faire, nous porterons une attention certaine à leur contenu : était-ce plutôt des livres politiques qui ne pouvaient être publiés ailleurs du fait de leur contenu compromettant? Si nous répondons par la positive, cela confirmerait la Belgique dans son image de pays d'édition libéral. Ces livres étaient-ils écrits en polonais ou en français? Et pour quel public? Servaient-ils à tourner en ridicule un régime en particulier? Ou s'agissait-il surtout de travaux plutôt inoffensifs, tels que des études historiques ou des ouvrages qui ressortaient des BellesLettres?

8 Les réponses apportées à ces questions nous en apprendront beaucoup sur les principales activités des émigrés politiques durant la période romantique: écrire, éditer et faire de la propagande. Cela nous permettra de créer une image correcte de la Belgique comme pôle attractif pour les publicistes, grâce à un certain degré de libéralisme qui la caractérisait alors, pensons par exemple à la garantie constitutionnelle de justice de jury pour des délits de presse ${ }^{11}$.

\section{Imprimeurs polonais}

9 Dès le début de la Grande Emigration, des Polonais tentèrent de créer leur propre imprimerie à Bruxelles. En septembre 1835, Konstanty Zaleski ouvrit une maison d'édition, mais il ne trouva ni l'argent ni le matériel pour réaliser une seule publication $^{12}$. Un an plus tard, Lelewel écrivit qu'il avait fondé une imprimerie. Il était prêt à éditer les ouvrages de ses compatriotes en France et leur demandait d'envoyer leurs manuscrits à Wiktor Tyszka ${ }^{13}$. On ne sait rien de ce qui se passa ensuite, mais le silence des sources laisse supposer un piètre succès. Les historiens polonais ont donc raison lorsqu'ils écrivent que la société fondée par Henryk Kałussowski, au milieu de 1837, était la première imprimerie polonaise à Bruxelles ${ }^{14}$.

10 Cette société, fondée dans le but de rompre le monopole dans le domaine de l'édition qu'avait Aleksander Jełowicki à Paris ${ }^{15}$, édita immédiatement trois magazines différents. Ziemianin Wszerada (Un cultivateur de bon conseil) était un périodique socioculturel dont les articles parlaient de mode vestimentaire, de marchandises et de machines. Son prospectus sortit de presse le 30 juillet. Sprawy emigracji (Les affaires de l'émigration), rédigé par Franciszek Gordaszewski, prêchait l'unification du camp démocrate à la veille de la création du ZEP (Union de l'émigration polonaise). Le deuxième cahier parut le 30 août. Quant à Naród Polski (La nation polonaise), il était auparavant édité à Paris, mais y fut interdit en automne 1836. Kałussowski n'en publia que le dernier numéro ; daté d'octobre 1836, celui-ci mais ne parut qu'à l'été de l'année suivante.

11 Kałussowski édita aussi des livres. On a trouvé des œuvres de Lelewel, de Karol Różycki et de Walenty Zwierkowski datées de 1837, et des œuvres de Michał Budzyński et de Jan Dworzecki datées de $1838^{16}$. Sa maison d'édition faisait également fonction d'entreprise de vente par correspondance, où l'on pouvait commander livres et manifestes par courrier ${ }^{17}$.

$12 \mathrm{Au}$ début, les affaires allaient bon train. Après quelques mois, un poste de deuxième collaborateur, aux côtés d'Adam Pausza, fut ouvert. Ce fut probablement Józef 
Kleczyński qui occupa cette fonction. La société déménagea du numéro 280 de la chaussée d'Ixelles au numéro 55 de la "rue Royale neuve " [sic ${ }^{18}$. Mais Kałussowski rencontra rapidement des difficultés. La collaboration espérée avec Poznań et Cracovie ne se réalisa pas. Afin de cacher un bilan négatif, il s'associa en janvier 1838 à l'imprimeur belge Lejeune. Mais quand celui-ci fit faillite fin mars (selon Kałussowski il s'agissait d'une faillite frauduleuse ${ }^{19}$ ) le sort de l'imprimerie de Kałussowski fut également scellé.

Kleczyński tenta de relancer la société, mais elle fut vendue en un mois ${ }^{20}$. Le rédacteur, Gordaszewski, dut faire paraître les derniers numéros de son Polacy na tułactwie chez un éditeur belge ${ }^{21}$. En juin 1838, Kałussowski quitta Bruxelles pour Londres, et se rendit aux Etats-Unis au mois d'août. Là-bas, il joua encore un rôle important dans la vie de la commaunauté émigrée. À Bruxelles, Tyszka fut chargé de la vente du stock, mais il réussit à grand-peine. Finalement, il transmit tous les papiers de Kałussowski à un nouvel imprimeur polonais à Bruxelles, Jan Nepomucen Młodecki²2 .

Młodecki avait déménagé de l'Angleterre vers la Belgique en 1835; il avait une expérience dans le monde du livre ${ }^{23}$. En 1837, il distribua en Belgique les produits issus de l'imprimerie et des librairies polonaises à Paris. En tant que membre actif de la gmina (communauté) bruxelloise, il s'impliqua en 1839 dans la création de la revue Orzet Biały, publiée à Bruxelles pendant huit ans. En tant qu'administrateur de ce périodique bruxellois (sous le pseudonyme de Monsieur Sarmata), il inséra, à partir de janvier 1840, des annonces destinées à promouvoir la vente d'une série de livres. Il n'est donc pas étonnant qu'il ait fondé, au mois d'août 1840 , une nouvelle imprimerie polonaise. Il l'installa au numéro 92 de la rue Léopold, et lui donna le nom «imprimerie belgofrançaise $»^{24}$.

Cette firme imprima l'Orzeł Biały à partir du 20 août 1840 et, à la fin de l'année, le rapport de l'Anniversaire du 29 novembre de cette année-là. Elle est également mentionnée sur la page de garde de deux livres qui furent probablement édités ailleurs ${ }^{25}$. L'imprimerie fut donc moins active que celle de Kałussowski en 1837-38. En septembre 1841, après un peu plus d'un an d'activité, elle dut elle aussi déposer son bilan et confier l'impression de l'Orzet Biały à un imprimeur belge : d'abord Bourlard et, à partir de novembre, J. H. Briard. La deuxième imprimerie polonaise n'avait donc pas existé très longtemps, elle non plus. Le fait que seul l'Orzeł Biały y ait été imprimé incite à se poser la question de savoir si cette société fut vraiment une imprimerie.

Młodecki déménagea au 56 de la rue de Ruysbroek et y continua ses activités dans le monde du livre, bien qu'il ne possédait d'imprimerie propre. Parallèlement, il poursuivait ses annonces dans l'Orzeł Biały destinées à promouvoir la vente de livres parus aussi bien en Belgique ou dans d'autres pays d'Europe de l'Ouest qu'à Leipzig et à Poznań. Il prit aussi soin de quelques éditions ${ }^{26}$.

17 Il continua également à suivre l'édition de publications polonaises comme Orzeł Biały et les rapports des commémorations du 29 novembre (Anniversaires). Lelewel écrivit au sujet du rapport du 29 novembre 1844 (imprimé chez Briard) que « Młodecki a retardé l'impression d'une ou deux semaines $»^{27}$. Młodecki sous-traitait donc le travail aux imprimeurs belges. La supposition de son biographe, Andrzej Kłossowski, qu'il ait été responsable de la majeure partie des publications polonaises en Belgique semble erronée $^{28}$. On ne peut pas expliquer, par exemple, pourquoi le nom de Młodecki ne figurait que sur quelques livres et non sur la majorité d'entre eux. 

«Młodecki était ici le seul à s'occuper d'une librairie générale ${ }^{29}$. C'était sans doute le cas en 1843 , mais au début des années 1840 , il y avait une autre librairie polonaise à Bruxelles. Elle fut exploitée par Andrzej Szmigielski, déjà présent à Bruxelles en 1833. Il ne dut lancer sa drukarnia polska (imprimerie polonaise) qu'à la fin des années 1830 . On connaît quatre ouvrages imprimés chez Szmigielski ${ }^{30}$. Il fut probablement également impliqué dans Antiquité de Pologne de Lelewel paru en $1842^{31}$. En 1845, ce dernier le mentionnait comme compositeur en Belgique ${ }^{32}$.

Szmigielski travailla probablement chez un imprimeur belge, et c'est grâce à cela qu'il réussit à imprimer quelques ouvrages sous l'enseigne de sa drukarnia polska. On ne peut toutefois pas confondre celle-ci et l'Imprimerie belgo-française de Młodecki. Kłossowki, le biographe de Młodecki, se trompe lorsqu'il suppose (car aucune source n'est disponible) que Młodecki fut également impliqué dans les éditions de la drukarnia polsk $a^{33}$. Si l'on considère une perspective plus large, il est surtout important de retenir que la société de Szmigielski ne tint pas longtemps, elle non plus.

Après 1846, il n'y eut plus un seul imprimeur polonais en Belgique durant plus d'une décennie. Vers 1858, Zygmunt Gerstmann s'installa en Belgique. Il eut d'abord une librairie à la rue Neuve, à Bruxelles (où Wiktor Heltman avait travaillé quelque temps) ${ }^{34}$. En 1860, ou peu avant, il acheta les tirages de la Librairie étrangère de J. N. Bobrowicz, en liquidation à Leipzig. Il réimprima les œuvres en les dotant d'une nouvelle page de garde, d'une nouvelle année, d'un nouveau lieu d'édition et y plaça son nom.

21 Gerstmann imprima bien quelques œuvres d'auteurs polonais de Belgique, ${ }^{35}$ mais la majorité de ses publications furent des réimpressions d'œuvres littéraires polonaises parues à Leipzig. Il poursuivit la vente de séries entamées par Bobrowicz, comme la Biblioteka Powieści Historycznych (Bibliothèque de récits historiques) et la Biblioteka Malownicza Najbawniejszych Powieści dla Dzieci po francusku i po polsku (Bibliothèque pittoresque des plus amusantes histoires pour enfants en français et en polonais). Simultanément, il démarra une nouvelle série des tirages de Leipzig: la Biblioteka Domowa (Bibliothèque de la maison). Il essaya de promouvoir la vente en baissant radicalement les prix et en ouvrant une nouvelle librairie à Ostende (au numéro 18 de rue de Flandre), ouverte uniquement pendant la saison touristique. Mais ses affaires ne furent guère brillantes. Gerstmann liquida sa société en 1865 et partit vraisemblablement à Berlin ${ }^{36}$.

Seul le cinquième imprimeur polonais de Belgique, Henryk Merzbach, eut une société florissante. Merzbach descendait d'une famille d'imprimeurs de Varsovie. Son père Zygmunt et son oncle Samuel ouvrirent, dans les années 1830, une librairie dans la capitale polonaise, et lui-même y posséda, dès 1860, une maison d'édition située dans la rue principale, Krakowskie Przedmieście, maison qui fut fermée en 1863 par les Russes. Fin août 1864, Merzbach s'installa à Bruxelles, où il fut immédiatement embauché (ce qui laisse penser que ceci avait donc été fixé avant son arrivé) dans la prestigieuse Librairie européenne de Charles Muquardt, située sur la place Royale. Au mois d'octobre de la même année, il en assuma la direction générale ${ }^{37}$.

Initialement, Merzbach était fort enthousiaste à l'idée de faire de la maison Muquardt une société d'éditions polonaise. Il écrivit en ce sens quelques lettres à Józef Ignacy Kraszewski afin de convaincre cet auteur et éditeur de déplacer ses activités de Leipzig en Belgique, mais ce projet ne se concrétisa pas $^{38}$. Quand la société fut mise en vente 
trois ans plus tard, il essaya, auprès de compatriotes comme Kraszewski, Aleksander Kurtz et le comte Wielhorski, de rassembler des fonds pour racheter l'imprimerie et la transformer en un "office de publicité européenne" polonais. L'achat eut effectivement lieu. Début 1869, on pouvait lire sur les en-têtes la mention "Henry Merzbach Succ. ». Sept ans plus tard, Merzbach se nommait même « libraire de la Cour et de S. A. R. le Comte de Flandre $»^{39}$.

Il n'y avait probablement pas d'investissements polonais dans cette nouvelle firme. Elle n'avait ni connections explicitement polonaises, ni de profil polonais. La maison Muquardt, qui avait, avant 1864, encore publié l'une ou l'autre œuvre polonaise ${ }^{40}$, ne le fit plus sous la direction de Merzbach (sauf quelques exceptions en 1868 et $1878^{41}$ ). En 1883, Merzbach écrivit explicitement à un collègue de Cracovie qu'il n'éditait ni brochures, ni œuvres polonaises, car celles-ci n'étaient ni lues ni vendues en Belgique ${ }^{42}$. Il avait déjà avoué, en 1876, son engagement minime pour la Pologne, sur un ton quelque peu littéraire : « Le pin polonais se dessèche à l'étranger. " ${ }^{43}$

La raison pour laquelle Merzbach eut du succès et pour laquelle les autres éditeurs polonais échouèrent est claire. Merzbach était un éditeur belge, se concentrant sur le marché belge ou ouest-européen. Ses compatriotes, au contraire, ne pouvaient survivre en n'éditant que des œuvres polonaises. Publier en exil s'avérait non lucratif, ni pour l'éditeur, ni pour l'auteur.

En réalité, la majorité des œuvres polonaises publiées en Belgique le furent par des éditeurs belges. Dans les années 1830, Prosper Voglet, installé sur le boulevard de l'Empereur, fut l'imprimeur le plus important des Polonais à Bruxelles. Il avait des sympathies démocrates radicales (il avait, par exemple, imprimé La Voix du Peuple d'avril à juin 1833) ${ }^{44}$, il disposait de caractères typographiques polonais et il employa durant un certain temps Tadeusz Idzikowski ${ }^{45}$.

Dans les années 1840, Briard (au numéro 34 de la rue des Six Jetons) repris ce rôle, et mis sous presse la plupart des Anniversaires et l'Orzet Biały ${ }^{46}$. La majeure partie des titres, tous parus entre 1851 et 1869 , furent publiés par J. H. Dehou, le beau-frère de Jacob Kats $^{47}$, qui imprima également les périodiques Demokrata Polski, Wytrwałość et Ognisko. Après 1850 , Polska et La Pologne furent les seuls périodiques polonais réalisés ailleurs que chez Dehou, notamment chez J. Nys. Ce dernier édita pendant l'insurrection de Janvier quelques œuvres polonaises. Notons encore que Ludwik Ozeasz Lubliner publia régulièrement chez $\mathrm{Ch}$. Vanderauwer ${ }^{48}$.

\section{Auteurs polonais}

28 Il y avait beaucoup plus d'auteurs polonais que d'imprimeurs en Belgique. La liste, basée sur la Bibliografia polska XIX stulecia de Karol Estreicher, des ouvrages écrits par

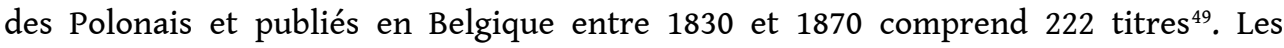
œuvres sont bien réparties dans le temps : 35 datent des années 1830, 59 des années 1840, 32 des années 1850 et 95 des années 1860. Ce dernier nombre s'explique par l'activité de l'imprimerie de Gerstmann : sur la seule année 1862, 35 livres avaient paru, la plupart étant des réimpressions.

29 Evidemment, tous les auteurs n'habitaient pas en Belgique. La liste contient par exemple des livres de Mickiewicz, de Leonard Chodźko et de Jan Czyńskij ${ }^{50}$. Inversement, certains auteurs polonais de Belgique publiaient aussi à Paris ou à Leipzig. 
Des vingt-cinq œuvres écrites par Lelewel entre 1833 et 1861, seules huit furent éditées là où il vivait, à savoir à Bruxelles. Il en publia six à Poznań, cinq à Paris, deux à Leipzig, deux à Varsovie et une à Rouen ${ }^{51}$.

La grande majorité des livres traitaient de sujets qui n'étaient pas liés à la politique de l'époque ; il s'agissait surtout d'œuvres littéraires ou historiques. Lelewel était l'auteur le plus prolifique (et le plus revu et le plus réimprimé). Il publia principalement des études sur la numismatique et sur la géographie historique (dont la Numismatique du Moyen Age [1835] et la Géographie du Moyen Age [1849] sont les plus importantes), ainsi que des synthèses de l'histoire polonaise : Dzieje Polski potocznym sposobem (Histoire de la Pologne au quotidien, 1837), Polska odradzająca siĘ (La Pologne ressuscitant, 1836) et Panowanie króla [...] Poniatowskiego (Le règne du roi Poniatowski, 1847).

31 Quelques autres Polonais publièrent en Belgique des ouvrages d'histoire générale polonaise ou sur certains thèmes de l'histoire d'Europe occidentale : hormis Chodźko et Czyński, citons par exemple Heltman, Tespezjusz Dubiecki et Leon Leopold Sawaszkiewicz ${ }^{52}$. Quelques œuvres comportaient un message politique explicite (Sawaszkiewicz écrivit formellement en 1859 qu'il comparait l'expédition à Moscou de Stanisław Żółkiewski [1610] à celle de Napoléon [1812] parce que « le passé est l'oracle du futur ») mais dans la plupart des cas, la valeur de l'analyse résidait essentiellement dans la création de nouvelles visions de l'histoire polonaise et dans le maintien de l'identité polonaise ${ }^{53}$.

32 Il en allait de même pour les œuvres littéraires. Certains Polonais publièrent en Belgique de la prose et de la poésie : Dworzecki, Budzyński, Merzbach, Jan Ignacy Moll, Jan Kanty Radecki (qui n'habitait pas en Belgique), Czesław Karski et Włodzimierz Wolski ${ }^{54}$. Leur nombre reste fort limité et leurs œuvres de qualité moyenne. Il s'agit d'auteurs de second ordre, n'atteignant pas le niveau de leurs compatriotes en France qui élevèrent le romantisme après 1830 à l'un des points culminants de la littérature polonaise. Ce n'est qu'au début des années 1860 qu'une plus grande quantité d'œuvres littéraires furent éditées, grâce à Gerstmann.

33 Ce grand nombre d'ouvrages historiques et littéraires ne signifie pas qu'il n'y avait pas de publications liées à la vie politique. Cependant, celles-ci traitaient surtout de la politique interne, c'est-à-dire purement polonaise. On trouve par exemple des comptes d'organisations, des manifestes, des polémiques sur des membres du comité du ZEP, des programmes ou encore des rapports de manifestations et de célébrations, des périodiques, etc. On pourrait également considérer les cinq livres d'éducation militaire comme des publications politiques internes ${ }^{55}$.

Un autre genre proche de la politique était la polémique. En effet, les discussions entre exilés étaient régulièrement débattues par écrit, surtout sur des thèmes précis. En 1837, Różycki publia par exemple ses commentaires sur l'expédition que le général Józef Dwernicki mena en Ruthénie en 1831, puis, l'année suivante, une réponse à une série d'articles publiés en France ${ }^{56}$. Jan Nepomucen Umiński réagit, en 1843, à l'article d'un certain Chotomski sur les événements de fin septembre 1831 à Słupno et à Płock ${ }^{57}$. En 1855, Józef Kalasanty Godebski répondit à Walery de Rottermund, concernant ses objections au rapprochement de Maciej Rybiński et de Napoléon III et à l'espoir des monarchistes de mener une grande guerre pour laquelle ils auraient le soutien de l'empereur français ${ }^{58}$. Le titre de son livre, Ah! L'honnête homme, évoque parfaitement le style de ces polémiques. Les attaques personnelles reflètent l'étroitesse d'esprit de la communauté exilée. 

situation polonaise. En 1832, Bonawentura Niemojowski et Michał Pietkiewicz, représentants du Sejm (diète polonaise) habitant cette année-là quelques mois à Bruxelles, écrivirent chacun un livre en français sur l'insurrection polonaise ${ }^{59}$. En 1838, Budzyński, arrivé l'année précédente d'Angleterre, fit de même au sujet de son emprisonnement en Galicie entre 1833 et $1836^{60}$. Umiński publia en 1844 Gedichte des polnischen Aufstandes (Poème de l'insurrection polonaise), en allemand. La même année, deux livres critiquèrent la situation polonaise : celui de Lelewel à propos de la politique d'enseignement en Lituanie en 1824 (l'année où il fut renvoyé de l'Université de Vilnius), et celui de Dubiecki avec un poème sur les années 1830 et 1840 dans sa patrie ${ }^{61}$. Ils ne purent cependant pas inverser la tendance générale : les Polonais de Belgique publiaient peu sur la situation dans leur pays dans les langues d'Europe occidentale.

(1846, on publia trois livres en français sur la Pologne : un discours sur l'occupation autrichienne de Cracovie, de la plume de Dubiecki; l'histoire de Makryna Mieczysławska (abbesse à Minsk); et l'ouvrage d'un Cracovien, W. Wolniewicz, sur la révolte qui éclata cette année-là dans la ville $^{62}$. En 1847, parut le plaidoyer de Ludwik Mierosławski au procès de Berlin contre les prisonniers de $1846^{63}$. En 1848, sortirent deux réflexions sur la situation en Pologne ${ }^{64}$. Et en 1849 , Hipolit Terlecki fit paraître un récit de sa détention à Dresde en mai de cette même année ${ }^{65}$.

Par la suite, la propagande et les écrits d'information en langues d'Europe occidentale cessèrent à nouveau d'être publiés. Ceci est assez paradoxal. En effet, on s'attendrait à ce que les proscrits veuillent rappeler leur sort au monde occidental, sort menacé de tomber dans l'oubli.

En Belgique, Lubliner fait exception. Ce Juif polonais décrivit la situation inadmissible qui régnait en Pologne également à des moments plus calmes. Sa formation juridique Lubliner avait étudié le droit à l'ULB pour devenir ensuite avocat à la Cour d'appel de Bruxelles - se manifeste dans ses écrits. En 1840, il étudia le droit civil (renouvellé en 1832) et la législation matrimoniale en Pologne, et en 1850 il examina de près le nouveau (depuis 1847) code pénal en Pologne.

39 En 1855, désapprouvant les mesures d'amnistie du nouveau tsar Alexandre II, il publia une étude sur la politique de confiscation de Nicolas I ${ }^{\mathrm{er}}$, père de ce dernier, et souligna l'antisémitisme qui régnait en Pologne russe. Ces publications dénotent parmi les autres titres politiques en langues d'Europe occidentale. Elles furent écrites à d'autres moments que durant l'insurrection, et traitèrent d'autres développements (contrairement aux mémoires ou aux histoires des insurrections).

La deuxième raison pour laquelle Lubliner fait figure d'exception parmi les auteurs en exil est le fait que ses études paraissaient également en polonais, elles furent donc lues en Pologne et même commentées dans la Gazeta Warzawska ${ }^{66}$. Lubliner fut l'un des seuls Polonais de Belgique, avant 1860, à s'adresser à l'ensemble des Polonais par ses écrits. Ses points de vue alimentèrent des polémiques dans la presse de Varsovie. L'explication de cette situation exceptionnelle est simple : il réagissait à des situations intolérables très précises, comme l'antisémitisme. Tous les autres ouvrages politiques publiés en polonais en Belgique traitaient uniquement de situations internes à l'exil ou de projets d'unification, ce dont la Pologne n'avait que faire. 

Belgique), quatre histoires dans Kłosy, trois histoires dans Dziennik Literacki et autant dans Tygodnik Ilustrowany, deux histoires dans Strzecha et une histoire dans Przegląd Tygodniowy, dans Tygodnik Romansów i Powieści et dans Niwa (dans la majorité des périodiques littéraires de la Pologne russe). En outre, trois récits furent édités sous forme de romans: Helena (Varsovie, 1867), Siostrzane dusze (Varsovie, 1872) ${ }^{76}$ et Opowiadanie Stasia (Le récit de Stanisław, Lviv). Certaines œuvres furent même traduites en russe : Helena en 1878 à Varsovie, et d'autres romans à Saint-Pétersbourg en 1871 et en 1874. Toutes étaient des œuvres de fiction, avec de temps en temps une petite 
référence à l'actualité (par exemple dans l'histoire intitulée Nihilista [Le Nihiliste]). Seule la biographie de Mierosławski fut un sujet plus délicat, et Miłkowski la publia à Paris.

Pour les historiens belges, c'est surtout l'ouvrage de Teodor Tomasz Jeż intitulé Belgija $i$ Belgowie (La Belgique et la Belges) qui attire l'attention. Ce feuilleton, qui parut dans Niwa en 1872, traite de l'histoire des Pays-Bas méridionaux ${ }^{77}$. Il contient surtout des faits bruts, c'est pourquoi les Listy z Belgii (Lettres de Belgique) sont plus intéressantes pour l'analyse des images polonaises de la Belgique ${ }^{78}$. Ces lettres de fiction furent publiées en 1878 et 1879 dans le Kurier Warszawski par Merzbach et Wolski. Avec Miłkowski et peut être aussi Władysław Sabowski ${ }^{79}$, ces deux hommes étaient les seuls Polonais installés en Belgique à avoir publié dans la presse polonaise (bien que d'autres Polonais de Belgique aient eu des contacts avec, par exemple, la maison d'édition de Kraszewski à Dresde) ${ }^{80}$.

Tout comme Wolski, Merzbach publia surtout de la poésie ${ }^{81}$, mais ses contacts avec le monde d'éditeurs en Pologne allèrent plus loin. En 1879, il partagea son expérience avec un Polonais qui avait démarré une imprimerie à Varsovie après un exil de plusieurs années à Krasnoïarsk, en Sibérie ${ }^{82}$. En 1889, Merzbach demanda une nomination officielle (et légalisée par le consul belge) comme collaborateur du Kurier Warszawski ${ }^{83}$. La frontière entre la patrie polonaise et l'exil était à cette époque déjà très mince.

\section{Conclusions}

Cet article avait pour but d'analyser la place de la Belgique en tant que centre d'édition et d'impression pour les émigrés polonais de la période romantique. On peut résumer la situation par les chiffres suivants. Cinq imprimeries et maisons d'éditions furent créées et exploitées à Bruxelles par des exilés polonais : par Kałussowski en 1837-38, par Szmigielski vers 1839-1840, par Młodecki dans les années 1840, par Gerstmann entre 1860 et 1865, et par Merzbach à partir de 1864 . On ne peut toutefois pas parler de succès. Kałusskowski et Szmigielski firent rapidement faillite, Młodecki sous-tirait quant à lui son travail aux imprimeurs belges. Gerstman publia des dizaines d'ouvrages, mais ceux-ci étaient principalement des réimpressions d'œuvres parues auparavant à Leipzig. Seul Merzbach eut une affaire fleurissante, mais c'était une maison d'édition belge, la maison Muquardt, qui publiait des livres pour un public belge. Le marché pour les livres polonais était trop petit. Même la librairie de Gerstman à Ostende, où de nombreux aristocrates polonais s'installaient pourant le temps des vacances, dut rapidement fermer ses portes.

50 La plupart des auteurs polonais publiaient leurs travaux auprès d'éditeurs belges. $\mathrm{Au}$ total, entre 1830 et 1870, plus de 200 œuvres furent ainsi publiées en Belgique par des auteurs polonais. Ce qui ne fait pas de la Belgique un centre international d'édition pour la communauté émigrée. De nombreux Polonais installés en Belgique, Joachim Lelewel par exemple, publiaient leurs livres à l'étranger. La plupart des œuvres qui apparaissaient sur le territoir de la Belgique étaient dépourvues de caractère compromettant. Il s'agissait plutôt d'études historiques, de travaux littéraires, de discussions sur la vie associative des émigrés, etc. Leurs publications n'avaient rien de politique, et le fait de publier en Belgique n'était pas dicté par des raisons politiques. Ce n'est que durant des périodes précises, surtout en 1846-1848 et 1863-1864, que les 
Polonais écrivaient des livres pour informer les lecteurs belges ou ouest-européens sur la situation de leur pays. Il y a bien sur des exceptions, comme par exemple Lubliner, qui publia des pamphlets politiques, aussi bien en polonais qu'en français. Dans la seconde moitié des années 1860, quelques Polonais émigrés en France firent sortir leurs livres en Belgique, pour contourner la censure qui régnait sous le second Empire. Il ne faut toutefois pas exagérer leur impact. A aucun moment les réfugiés polonais ne se référèrent à une possible réputation libérale d'une législation progressiste sur la presse en Belgique. D'autant plus que de nombreux auteurs écrivaient en polonais dans des périodiques polonais. Ces éléments concerant les émigrés polonais de la période romantique doivent être mis en coprésence pour édifier une image correcte de la situation du domaine de l'édition en Belgique. La réponse est sans appel : le pays n'était pas un paradis de l'édition pour les émigrés, et tous les migrants polonais de la période romantique qui se trouvaient en Belgique ne publiaient pas travaux ayant pour but la défense de leur patrie.

\section{NOTES}

1. Vandersteene L., « De mythe van het gastvrije België. Belgisch nationalisme en herinneringen aan een gastvrij verleden in de negentiende eeuw en vandaag» (Le mythe de la Belgique hospitalière. Le nationalisme belge et les souvenirs d'un passé hospitalier au XIX siècle et aujourd'hui), in: Handelingen van de Koninklijke Zuid-Nederlandse Maatschappij voor Taal- en Letterkunde en Geschiedenis, vol. LIV, 2000, pp. 315-334 ; Goddeeris I., « Belgische pull-factoren bij politieke vluchtelingen. Een perceptie vanuit de longue durée " (Les facteurs d'attraction belges chez les réfugiés politiques. Une perception de longue durée), in : Ibid., pp. 271-289.

2. Billen Cl. (ULB), «Kruispunt van culturen » (Carrefour de cultures), in : Brussel. Kruispunt van culturen (Bruxelles, carrefour des cultures), Hoozee Robert (dir.), Mercatorfonds, Antwerpen, 2000, p. 24. A propos de la loi des étrangers à laquelle elle fait référence : Vandersteene L., « Het uitleveringsrecht en de bescherming van politieke vluchtelingen in het negentiende-eeuwse West-Europa, met bijzondere aandacht voor Nederland en België » (La loi sur l'extradition et la protection des réfugiés politiques au $\mathrm{XIX}^{\mathrm{e}}$ siècle en Europe occidentale, avec une attention particulière pour les Pays-Bas et la Belgique), in : Pro Memorie, vol. II, n² 2, 2000, pp. 256-272.

3. Stengers J., Emigration et immigration en Belgique au XIX ${ }^{e}$ et au XX $X^{e}$ siècles, Académie royale des Sciences d'Outre-Mer, Bruxelles, 1978, p. 73.

4. Belgique, terre d'accueil? Rejet et accueil des exilés politiques en Belgique de 1830 à nos jours, Morelli A. (dir.), actes du Colloque "L'émigration politique en Europe aux XIX et XX ${ }^{\mathrm{e}}$ siècles.», 3-5 mars 1988, Rome, Ecole française de Rome, Rome, 1991, p. 119. Un an plus tard, Herbert Reiter qualifiait lui aussi de restrictive la politique d'émigration belge. Voir : Reiter H., Politisches Asyl im 19. Jahrhundert. Die deutschen politischen Flüchtlinge des Vormärz und der Revolution von 1848/49 in Europa und den USA (L'asile politique au XIX ${ }^{e}$ siècle. Les réfugiés politiques allemands de l'avantmars et de la Révolution de 1848-1849 en Europe et aux États-Unis), Duncker und Humblot, Berlin, 1992, pp. 116-118.

5. Sartorius Fr., "Autour de Marx, Bruxelles, 1847-1848, les membres du Deutscher ArbeiterVerein ", in: Mélanges offerts à Claire Dickstein-Bernard, Bonenfant P. et Cockshaw P. (dir.), Bibliothèque royale Albert $\mathrm{I}^{\mathrm{er}}$, Bruxelles, 1999, pp. 366-367. 
6. Quaghebeur M., "Een toevluchtsoord voor schrijvers en filosofen » (Un havre pour les écrivains et les philosophes), in: Brussel. Kruispunt van culturen, op. cit., pp. 36-43; SaintFerréol A., Les proscrits français en Belgique ou La Belgique contemporaine vue à travers l'exil, Godet, Paris, 1875, p. 226 et ss. et p. 252 et ss.

7. Goddeeris I., «Van favoritisme naar legaliteit: de Belgische tolerantiedrempel voor politieke activiteiten van ballingen, 1830-1914» (Du favoritisme à la légalité. Le seuil de tolérance belge pour les activités politiques des exilés), in : Belgisch Tijdschrift voor Nieuwste Geschiedenis, vol. XL, n० 3, 2010, pp. 313-344.

8. Ronin Vl., "Le publicisme russe en Belgique au milieu du XIX ${ }^{\mathrm{e}}$ siècle », in : Revue des Pays de l'Est, vol. XXXII, n 1-2, 1991, pp. 1-39.

9. Goddeeris I., «Belgique - terre d'accueil: perceptie en attractiviteit van België als gastland bij Poolse politieke migranten (1831-1846)» (Belgique, terre d'accueil : perception et attractivité de la Belgique en tant que pays d'accueil chez les émigrés politiques polonais [1831-1846]), in: Belgisch Tijdschrift voor Nieuwste Geschiedenis, vol. IXXX, n³-4, 1999, pp. 261-314.

10. Goddeeris I., «Ostatni uchodźcy romantyczni czy pierwsi emigranci ekonomiczni? Polska emigracja w Belgii z lat sześćdziesiątych XIX wieku» (Les derniers réfugiés romantiques ou les premiers émigrés économiques ? L'émigration polonaise des années soixante du XIX ${ }^{\mathrm{e}}$ siècle), in : Emigracja postyczniowa 1863 roku (L'émigration polonaise d'après janvier 1863), Niebelski E. (dir.), Wydawnictwo KUL, Lublin, 2010, pp. 103-138.

11. Delbecke Br., De lange schaduw van de grondwetgever. Perswetgeving en persmisdrijven in België, 1831-1914 (L'ombre étendue du législateur. La législation sur la presse et les délits de presse en Belgique, 1831-1914), Academia Press, Gent, 2012.

12. Lelewel J., Listy Emigracyjne (Lettres d'émigration), WiĘckowska H. (éd. et introduction), I 363 (20/09/1835) et I 374 ( \pm 14/10/1835), Nakł. Polskiej Akademii UmiejĘtności, Cracovie, 1848.

13. Ibid., II 81-82 (19/10/1836), Cracovie, 1848.

14. Voir e.a. :Pfeiffer-Milerowa Halina, « $Z$ historii księgarstwa. Lelewel i jego wydawcy» (De l'histoire du monde du livre. Lelewel et ses éditeurs), in : KsiEgarz, vol. XXX, n 4, 1986, pp. 13-14 ; Śladkowski W., Słownik pracowników książki polskiej (Dictionnaire des travailleurs du livre polonais), Nakładem Ministerstwa szkolnictwa wyższego, Varsovie-Łódź, 1972, pp. 388-389.

15. Lettre de Kałussowski $\mathrm{H}$. à Gadon $\mathrm{L}$ (03/08/1838, Londres), Bibliothèque nationale de Varsovie, $\mathrm{n}^{\circ} 8925,7 \mathrm{v}$. Dans cette lettre, Henryk Kałussowski consacre quelques pages à l'histoire de son imprimerie (à l'époque déjà liquidée). Celle-ci contient divers détails inconnus des chercheurs polonais.

16. De Joachim Lelewel : Dzieje Polski ... potocznym sposobem opowiedział, $i$ do nich 15 krajobrazów skreślit... (L'Histoire de la Pologne... il l'a racontée au quotidien et en a rayé 15 paysages...), H. Kałussowski \& Cie, Bruxelles-Leipzig, 3e édition, 1837; O monetach błaznów i niewiniątek. Przekład z francuskiego Henryka Kałussowskiego (Des monnaies des bouffons et des innocents), traduit du français par H. Kałussowski, Bruxelles, 1837 ; Manifest Polaków znajdujących sį̨ w Belgii (Manifeste des Polonais se trouvant en Belgique), Bruxelles, 1837. De Karol Różycki : Uwagi nad wyprawa Jenerała Dwernickiego na Ruś (Remarques sur l'expédition du général Dwernicki en Ruthénie), H. Kałussowski, Bruxelles, 1837 ; Stowarzyszeni Polacy w Bruxelli do emigracyi polskiej (Les Polonais associés à l'émigration polonaise à Bruxelles), Bruxelles, 1837 ; Stowarzyszeni Polacy $w$ Brukselli do emigracyi polskiej [Odezwa wzglĘdem proponowania kandydatów do centralizacyi; na końcu podpisy: Kopczyński Piotr, Idzikowski Tadeusz, Terlecki Tadeusz itd.] (Les Polonais associés à l'émigration polonaise à Bruxelles [Appel concernant la propositions des candidats pour la centralisation; à la fin les signatures :Kopczyński Piotr, Idzikowski Tadeusz, Terlecki Tadeusz, etc.), H. Kałussowski \& Pausza, Bruxelles, 1837. De Walenty Zwierkowski : Maximum [Projekt względem nabywania ziemi $i$ oświecania siĘ ludu] (Maximum [Projet concernant l'acquisition de terre et l'instruction du peuple]), H. Kałussowski, Bruxelles, 1837. De Michał Budzyński : Cztery lata 1833-1836 w Galicyi 
austriackiéj przez jednego z wiĘźniów (Quatre ans 1833-1836 en Galicie autrichienne par un des prisonniers), H. Kałussowski, Bruxelles, 1838 ; Quatre années 1833, 1834, 1835, 1836 dans la Gallicie Autrichienne par un prisonnier polonais, H. Kałussowski, Bruxelles, 1838. De Jan Dworzecki : Poezje. Z $2^{\text {ma }}$ rycinami (Poésies. Avec deux estampes), H. Kałussowski, Bruxelles-Leipzig-Paris, 1838.

17. Annonce dans : Sprawy emigracji poszytami ogłaszane (Les affaires de l'émigration), vol. I, 1837, p. 189.

18. On retrouve déjà le nom de Pausza dans les premiers numéros de Naród Polski et Ziemianin Wszerada. Le nom de Kleczyński, quant à lui, n'apparaît que dans des sources plus tardives (e.a. dans Lelewel J., Listy emigracyjne, op. cit., II 193 [12/04/1838]), mais même sa biographe (et arrièrepetite-fille) ne parvient pas à établir précisément à quel moment il a rejoint l'entreprise : voir De Champeaux-Kleczyński D., Józef Kleczyński. Patriota polski XIX stulecia zapomniany i przywrócony pamiĘci (Józef Kleczyński. Un patriote polonais du XIX siècle oublié et rappelé à la mémoire), Towarzystwo naukowe katolickiego uniwersytetu Lubelskiego, Lublin, 1992, pp. 26-27). En ce qui concerne l'annonce de la vacance et le message concernant le déménagement : Sprawy emigracji poszytami ogłaszane, op. cit., vol. I, 1837, p. 189.

19. Lettre de Kałussowski H. à Gadon L., op. cit., 9v.

20. Lelewel J., Listy emigracyjne, op. cit., II 189-190 (12/04/1838) et 193 (30/04/1838).

21. Le dernier numéro est daté du 10 novembre 1838 (Polacy na Tułactwie, vol. I/II, 1838). Quelques mois plus tôt, en août, Lelewel mentionnait même Gordaszewski comme étant le seul imprimeur polonais de Belgique à posséder sa propre imprimerie ; il s'agissait peut-être là de ses activités de rédaction et de publication (Lelewel J., Listy emigracyjne, op. cit., II 215 [08/08/1838]).

22. Lettre de Tyszka W. à Kałussowski H. (03/12/1848, s.l. [Bruxelles]), Bibliothèque nationale de Varsovie, $\mathrm{n}^{\circ} 8923,83$.

23. Jan Nepomucen Młodecki a fait l'objet d'un long article biographique. Sauf avis contraire, je tire toutes mes informations de celui-ci : Kłossowski A, «Jan Nepomucen Młodecki, ksiEggarz i wydawca polski w Belgii » (Jan Nepomucen Młodecki, libraire et éditeur polonais en Belgique), in : Rocznik Biblioteki Narodowej, vol. XVII-XVIII, 1981-1982 (1986), pp. 230-231.

24. Probablement par analogie avec certaines autres imprimeries de Bruxelles. On connaît ainsi avec certitude l'existence en 1839 d'une «Imprimerie anglaise » au numéro 14 de la rue Montagne de la Cour (voir Skrzynecki J. Z., Bibliothèque polonaise de Paris, $n^{\circ} 533$, p. 346); en 1846, Leopold Leon Sawaszkiewicz publia un livre à la "Librairie luxembourgeoise». L’imprimerie de Młodecki portait également le nom « Librairie belgo-française ».

25. En 1840, sur un ouvrage de Jan Nepomucen Umiński intitulé Beleuchtung des Werkes: Geschichte des polnische Aufstandes und Krieges [Éclaircissement de l'œuvre : l'histoire de la révolte polonais et de la guerre] (à côté des mentions Berlin et Paris), et en 1841 sur une œuvre de Jan Czyński, Avenir des femmes (à côté des noms Dolin et P. Baudoin ; le dernier étant l'imprimeur du livre).

26. En 1843, d'une sorte d'almanach (Kalendarzyk emigranta na rok 1843 [Petit calendrier de l'émigré pour l'année 1843]) et d'une œuvre de Lelewel (Polska odradzajĄca siĘ czyli Dzieje polskie od r. 1795 potocznie opowiedziane, z 3 kartami. Wydanie drugie pomnożone przypiskami [La Pologne renaissante ou l'histoire polonaise à partir de 1795, racontée au quotidien avec trois cartes]) ; en 1844, de trois œuvres littéraires de Ryszard Wincenty Berwiński (Poezye. CzĘść IIga[Poésie. Deuxième partie]), Filaret Prawdowski [pseud. de Henryk Kamieński] (O prawdach żywotnych narodu polskiego [Des vérités vitales du peuple polonais]) et Jan Kanty Radecki (Dzieła wydane w Poznaniu pod kryptonymem Ja. Ka. Ra. oraz Poezye Mazura [Oeuvres publiées sous le cryptonyme Ja. Ka. Ra. et poésies de Mazurie]) ; et en 1847, deux autres œuvres de Lelewel (Panowanie króla polskiego Stanisława Augusta Poniatowskiego, obejmujĄce trzydziestoletnie usilności narodu podźwignienia siĘ, ocalenia bytu $i$ niepodległości, napisał ... Wydanie po szósty raz pomnożone, $z$ dołączeniem porównania $d w u$ powstań narodu polskiego $r$. 1794 i 1830-1831 [Le règne du roi polonais Stanisław August Poniatowski, comprenant trente ans d'effort du peuple polonais pour se 
soulever, sauver son existence et son indépendance] et Stracone obywatelstwo stanu Kmiecego [sic] $w$ Polsce. Wydanie drugie [La citoyenneté perdue de l'étant de paysan en Pologne]).

27. Lelewel J., Listy emigracyjne, op. cit., III 245 (01/01/1845).

28. Kłossowski A., art. cit.,p. 235 \& 239.

29. Lelewel J., Listy emigracyjne, op. cit., III 72 (29/09/1843). Voir pp. 224 et 235.

30. En 1839, un manifeste de la gmina (Gmina Polaków w Bruxelli do emigracji polskiej [La commune des Polonais à Bruxelles de l'émigration polonaise]) et un recueil de poèmes de Budzyński (Pierwiosnki. Poezye. [Primevères]); en 1841, un récit du même Budzyński (Wacław Rzewuski, fantazya z czasów powstania podolsko-ukraińskiego w $1831 \mathrm{r}$. [Wacław Rzewuski, fantaisie du temps de l'insurrection en Podolie et en Ukraine en 1831]) et un livret anonyme pour officiers (Powinności oficerów przeznaczonych do małej wojny, czyli wojny pomocniczej [Les devoirs des officiers destinés à la petite guerre, c'est-à-dire à la guerre auxiliaire]).

31. Antiquité de Pologne, de Lithuanie et de Slavonie expliquée. N. 1. Notice sur la monnaie de Pologne [Inserée dans la Pologne illustrée] (imprimé chez Baylet, mais la «librairie polonaise » figure également sur la jaquette).

32. Lelewel J., Listy emigracyjne, op. cit., III 293 (29/06/1845).

33. Kłossowski A., art. cit.,p. 231, n. 31 (au sujet des deux ouvrages de 1841). Dans la note 33 (concernant 1839), il se montre plus nuancé.

34. Łuczakówna H., Wiktor Heltman, Poznań, 1935, p. 243.

35. En 1861, "Inter eruditissimos orbis terrarum princeps", des nécrologies de Lelewel (Lelewel et Heltman \& Sawaszkiewicz); en 1862, le livret 0 kształceniu matek (Sur l'éducation de mères); en 1863, une carte des provinces orientales de la République des Deux-Nations et des écrits de Wiktor Heltman, Józef Kalasanty Godebski et Alfred Lasocki ; en 1864, à nouveau des pamphlets sur l'actualité de F.Pomian et de Bolesław Swierszcz. L'année suivante, il édita encore un livre, à nouveau de Swierszcz.

36. Śladkowski W., op. cit., p. 255.

37. Merzbach H. à Kraszewski J. I., Bibliothèque Jagellone de Cracovie, $n^{\circ} 6519$, p. 465 (circulaire de la veuve de Ch. Muquardt, J. Heine, s.l., 18 octobre 1864), citée partiellement dans le chapitre précédent. Charles Muquardt était un Allemand né à Berlin en 1813, propriétaire d'une librairie à Leipzig dont il ouvrit en 1837 une filiale à Bruxelles, et décédé en 1863 (voir Sartorius Fr., «Les Allemands en Belgique ", in : Histoire des étrangers et de l'immigration en Belgique de la préhistoire à nos jours, Anne Morelli (dir.), Couleur livres, Bruxelles, 2004, 181). Il est donc possible que Merzbach et Muquardt se soient rencontrés à Leipzig. Voir également: Ergetowski R., «Wrocławski epizod w życiu Henryka Merzbacha » (L'épisode de Wrocław dans la vie de Henryk Merzbach), in : Ze Skarbca Kultury, vol. L, 1990, pp. 49-65.

38. Lettre de Merzbach H. à Kraszewski J. I. (22, 28 et 29/11/1864, Bruxelles), Bibliothèque Jagellone de Cracovie, $n^{\circ}$ 6519, pp. 461-464 \& 470. Voir également : Zarzycka M., « Dzieje drukarni Józefa Ignacego Kraszewskiego, Drezno-Poznań» (Histoire de l'imprimerie de Józef Ignacy Kraszewski, Drezno-Poznań), in : Studia o KsiĄżce, vol. VI, 1976, p. 83.

39. Lettre de Merzbach H. à Kraszewski J. I. (lettres écrites entre le 28/08/1867 et le 09/03/1869 Bruxelles/Ostende, et 06/11/1876, Bruxelles), Bibliothèque Jagellone de Cracovie, $\mathrm{n}^{\circ} 6519$, pp. 494-504 et $432 \mathrm{a}$ [=532].

40. Lelewel en 1849 et 1859 ; Ludwik Ozeasz Lubliner et le pamphlet Finis Poloniae en 1861.

41. En 1868, un ouvrage de Józefat Bolesław Ostrowski ; en 1874, l'extrait *Un mot d'Histoire sur les Jésuites dans l'ancienne Pologne de J. T. Prawdzic (qui avait précédemment paru dans la Revue de Belgique) et, en 1878, *Sprawa polska jako wewnĘtrzna państwa rosyjskiego (La question polonaise comme interne à l'état russe). Voir Estreicher K., Op. cit.

42. Lettre de Merzbach H. à Buszczyński St. (27/04/1883, Bruxelles), Bibliothèque de l'Académie polonaise des Sciences, Cracovie, $n^{\circ}$ 2064/3, p. 388a. 
43. Lettre de Merzbach H. à Kraszewski J. I. (06/11/1876, Bruxelles), Bibliothèque Jagellone de Cracovie, $n^{\circ} 6519$, p. 432a [=532]. Citation : « sosna polska na obczyźnie usycha ».

44. La Voix du Peuple. Journal de la propagande et des intérêts polonais, $n^{\circ} 1 / 7(14 / 04 / 1833)$ et $n^{\circ} 1 / 15$ (09/06/1833). Dans ce dernier numéro, un autre imprimeur est mentionné pour la première fois, l'Imp. de la Voix du Peuple.

45. Lelewel J., Listy emigracyjne, op. cit., II 244 (20/01/1839). Tadeusz Idzikowski n'y a pas travaillé longtemps : en janvier 1838, il débutait comme tanneur (Ibid., IV 179 [21/01/1838]).

46. Tandis que l'Anniversaire de 1839 et les numéros de l'Orzeł Biały publiés entre le 01/12/1839 et la création de l'Imprimerie belge-française de Młodecki (1/19 [20/09/1840]) le furent par Prosper Voglet.

47. Kuypers J., Jan Pellering. Een vergeten redenaar en anarchist (Jan Pellering. Un anarchiste et orateur oublié), Ontwikkeling SM, Anvers, 1962, p. 25.

48. Les noms mentionnés ci-dessus sont ceux de tous les éditeurs belges dont on sait qu'ils ont publié plus d'un livre d'un exilé polonais. Seul J. Van Buggenhoudt (qui publiait en 1855 à Rottermund et 1862 à Gałęzowski) constitue une exception.

49. Estreicher K., Bibliografia polska XIX stulecia, Cracovie. La liste est reprise dans ma thèse de doctorat (Goddeeris I, « De verleiding van de legitimiteit: Poolse Exilpolitik in België 1830-1870 en 1945-1980 » [La tentation de la légitimité : l'exil politique polonais en Belgique en 1830-1870 et en 1945-1980], Katholieke Universiteit Leuven, Louvain, 2001. Thèse de doctorat non publiée), où je discute également la fiabilité de cette œuvre.

50. Mickiewicz A., Livre des pélerins polonais, traduit du polonais par le Cte Ch. De Montalembert suivi d'un Hymne à la Pologne par F. de La Mennais, Tircher, Bruxelles, 1834 ; Chodźko L. J. B., Tableau de la Pologne ancienne et moderne sous les rapports géographiques, statistiques, géologiques, politiques, moraux, lyriques, législatifs, scientifiques et littéraires, par Malte-Brun. Nouvelle édition entièremenent refondue, augmentée et ornée des cartes., 2 vol., Libraire parisienne e.a., Paris-Bruxelles, 1830 ; Chodźko L. J. B., Tableau de la Pologne ancienne et moderne ou histoire générale de ce pays sous tous les rapports d'apres Malte Brun. Nouvelle édition revue et ornée des cartes, Bruxelles, 1832 ; Czyński J., Colonisation d'Alger d'après la théorie de Charles Fourier [Extr. du Nouveau Monde], Bruxelles, 1840 ; Czyński J., Avenir des femmes, Dolin, libr.-commissionnaire, à la Librairie Belge-Française, imprimerie de P.Baudoin, Bruxelles, 1841 (mention plus haut);Czyński J., Józefat Bolesław Ostrowski i jego przekonania (J. B. Ostrowski et ses certitudes), Bruxelles, 1841 ;Czyński J., Histoire de Pologne, depuis les temps les plus reculés jusqu'à nos jours, sur un plan nouveau, avec six tableaux synoptiques des événements, des synchronismes, etc., rédigée sur un plan entièrement neuf, avec tableaux synoptiques d'histoire, de géographie, de littérature, etc. par Victor Boreau, 6e édition revue et augmentée. Cours compl. et méthod. d'hist. univ. à l'usage des établ. d'éduc.,Ad. Wahlen et Cie., Bruxelles, 1848 ; Czyński J., Monsieur Proudhon. Une complète ignorance vaut meux que les demiconnaissances. A Paris, au Bureau du Journal la Pologne, J. Nys, Bruxelles, 1864.

51. Nowy Korbut. Bibliografia Literatury Polskiej (Le Nouveau Korbut. Bibliographie de la Littérature polonaise), t. 8 : Romantyzm, Hasła osobowe K-O (Romantisme. Lettres K à O), Państwowy Instytut Wydawniczy, Varsovie, 1969, pp. 239-241, nn.46-70. A propos des éditeurs de Lelewel, voir également : Pfeiffer-Milerowa H., art. cit., pp. 7-20.

52. Dubiecki T., La ville d'Ath. Son antiquité, son origine slave, ses époques remarquables, ses archives communales, ses monuments et édifices publics, ses institutions du culte, d'instruction et de bienfaisance, J. H. Briard, Bruxelles, 1847 ; Heltman W., Association scientifique universelle. Projet, J. H. Dehou, Bruxelles, 1860 ; Heltman W., Akt uwłaszczenia ludu. Projekt (Acte d'affranchissement du peuple. Projets), Bruxelles, 1863 ; Heltman W., Tablice synoptyczne historyi polskiéj. Wiek XV (Tableaux synoptiques de l'histoire polonaise. Le XV siècle), Z. Gerstmann, Bruxelles, 1863 ; Heltman W., Insurrection des Polonais, Bruxelles, 1863 ; Sawaszkiewicz L. L., Tablice historyczno-geograficzne (Tableaux historico-géographiques), Bruxelles, 1838; Sawaszkiewicz L.L., Le génie de l'Orient commenté de ses monuments monétaires: Etudes historiques, numismatiques, politiques et critiques, sur le 
cabinet musulman de M. Ignace Pietraszewski (contenant 2683 médailles) accompagnées de plusieurs planches, A. Vandale et libr. luxembourgeoise, Bruxelles, 1846 ; Sawaszkiewicz L. L., Uchwały Gminy Bruxelskiej [Bruxella, 23 IV 1846] (Les résolutions de la commune de Bruxelles [Bruxelles, 23/04/1846]), s.1., 1846; Sawaszkiewicz L. L., Porównanie wypraw na MoskwĘ Żótkiewskiego i Napoleona rozważył "Przeszłość jest wyroczniĄ przyszłości" Platon (Comparaison des expéditions à Moscou de Żółkiewski et de Napoléon), Milikowski (J. H. Dehou),Bruxelles-Ostende-Paris, 1859.

53. A propos de la contribution de Lelewel à l'historiographie polonaise, voir e.a.: Skurnowicz J. S., Joachim Lelewel, history and creation of a Polish national tradition (Lelewel, histoire et création d'une tradition nationale polonaise), Wysokinska T. et Pirard St. (dir.), actes du Colloque "Joachim Lelewel à Bruxelles de 1833 à 1861 ", 17-18 avril 1986, Bruxelles, 1987, pp. 177-190 et Stanley J. D., "Joachim Lelewel ", in Nation and History. Polish Historians from the Enlightenment to the Second World War (Nation et histoire. Les historiens polonais depuis l'époque des Lumières jusqu'à la Deuxième Guerre mondiale), Brock Peter, Stanley John D. et Wróbel Piotr J. (dir.), University of Toronto Press, 2006, pp. 52-84.

54. Dworzecki J., Poezje, op.cit.; Moll J. I., La liberté en Belgique, Bruxelles, 1839 ; Budzyński M., Cztery lata, op. cit., ; Budzyński M., Quatre années, op. cit., ; Budzyński Michał, Wacław Rzewuski..., Drukarnia Polska, Bruxelles, 1841 (mentionné plus haut) ; Radecki J. K., Dzieła wydane w Poznaniu pod kryptonymem Ja. Ka. Ra. oraz Poezye Mazura, J. N. Młodecki, Buxelles-Poznań, 1844 (mentionné plus haut) ; Karski Cz., La crise. Poésies, Bruxelles, 1859 ; Merzbach H., Z wiosny (Du printemps), deuxième édition, Bruxelles, 1865 ; Merzbach H., Adieu à Michel Berend. Quelques mots sur la mission historique des peuples, Bruxelles, 1867 ; Merzbach H., Deux discours maçonniques par le F., Bruxelles, 1867 ; Merzbach H., Toast wygnańców $w$ rocznicĘ powstania styczniowego (Le toast des exilés à l'anniversaire de l'Insurrection de janvier), J. H. Dehou, Bruxelles, 1868 ; Wolski Wł., Promyki. Nowe poezye liryczne (Petites lueurs. Nouvelles poesies lyriques), J. H. Dehou, Bruxelles, 1869.

55. Lelewel J., Powinności oficerów..., Drukarnia Polska, Bruxelles, 1841 (mentionné plus haut) ; Hunersdorf L., Equitation allemande. Méthode la plus simple et la plus naturelle pour dresser le cheval d'officier et d'amateur, suivie d'un supplément pour l'instruction du cheval de troupe et de son cavalier, traduite sur la $6^{e}$ édition (1840) par Armand de Brochowski capitaine commandant d'escadron au $1^{\text {er }}$ lancier belge, Bruxelles, 1843 ; Godebski J. K., Géométrie du jalon ou l'art de résoudre les problèmes usuels de géométrie pratique, à l'aide de simples alignements; contenant de plus la théorie élémentaire des transversales rectilignes, ainsi que la description des instruments et des moyens ordinaires pour tracer et mesurer des lignes droites. Ouvrages consacré à la pratique, J. H. Briard, Bruxelles, 1845 ; Racquillier F., Aphorismes militaires pour la guerre des Partisans (Guerillas) par ... ancien officier de l'armée francaise et de l'armée polonaise, V. Wouters, Bruxelles, 1850 ; Wilczyński Wł. St., Taktyka jazdy przez wyższego oficera wojsk polskich, z 3 tabl. litogr. (Tactiques d'équitation par un officier supérieur de l'armée polonaise),Bruxelles-Berlin, 1852.

56. Różycki K., Uwagi..., op. cit.; Różycki K., Odpowiedź na rozbiór siedmiu artykułów "PamiĘtnika Emigracyi" ogłoszony (Réponse à l'analise dans sept articles du PamiĘtnik Emigracyi), Bruxelles, 1838.

57. Umiński J. N., Kilka słów o zaszłych wypadkach w Słupnie i Płocku w d. 23 września 1831 r. wywołane przez pismo podpułkownika Chotomskiego "Odpowiedź Chełmickiemu i Zwierkowskiemu" (Quelques mots sur les accidents survenus à Słupno et à Płock le 23 septembre 1831), Bruxelles, 1843.

58. [Godebski J. K.], Ah! l'honnête homme. Réponse à M. Walery de Rottermund, Illustre seigneur de Gurna Klecza ; membre de diverses académies et sociétés d'agriculture; soi-disant auteur de la Réponse à la lettre du général Rybinski; de l'Appel aux véritables intérêts de la cause polonaise; de Napoleon III, le général Rybinski et Lord John Russel, etc. etc., Ch. Vanderauwer, Bruxelles, 1855.

59. Niemojowski B., L'autocrate et la constitution du royaume de Pologne, E. Laurent, Bruxelles, 1832 ; Pietkiewicz M., La Lithuanie et sa dernière insurrection, H. Dumont, Bruxelles, 1832.

60. Budzyński M., Cztery lata..., op. cit. 
61. Lelewel J., Novosiltzow à Vilna, ou Guerre impériale avec les enfants et l'instruction. Episode historique de 1824, J.H. Briard, Bruxelles, 1844 ; Dubiecki T., Wiesczby [sic]. Poemat z przydarzeń ojczystych IV $i V$ dziesiątka XIX stolecia. Napisał na wygnaniu ... (Des prophéties. Poème tiré des événements qui eurent lieu dans la patrie durant les $4^{\mathrm{e}}$ et $5^{\mathrm{e}}$ décennies du XIX ${ }^{\mathrm{e}}$ siècle. Ecrit en exil.), J. H. Briard, Bruxelles, 1844.

62. Dubiecki T., Les Autrichiens à Cracovie. Discours prononcé à Bruxelles le 29 novembre 1846 à l'occasion de la commémoration de la révolution polonaise, C.G.Vogler, Bruxelles, 1846; Mieczysławska M., Récit de Makrena Mieczysławska abtesse des Basiliennes de Mińsk ou histoire d'une persécution de sept ans soufferte pour la loi par elle et ses religieuses écrite sous sa dictée et d'après les ordres de N.T.S. Père le P. Gregoire XVI, par R. S. Maximilien Ryłło Recteur de la Propagande à Rome, l'abbé Alex. Jełowicki Recteur de l'Eglise de St. Claude à Rome, l'abbé Alois Leitner, Théologien de la Propagande à Rome, précédé d'une préface par Mr. le Mis de Beauffort, commencé le 6 novembre et terminé le 6 décembre 1845 dans le Convent de la Trinité à Rome. $3^{e}$ édition, C. G. Vogler, Bruxelles-Louvain, 1846 ; Wolniewicz W. A., Die Polnische Frage mit Bezug auf die letzten Ereignisse von einem Krakauer [La question polonaise en référence à l'expérience récente d'un Cracovien], C.G. Vogler, Bruxelles, 1846.

63. Mierosławski L., Procès des Polonais. Discours prononcé le 5 août 1847 devant la Cour crim. de Berlin [Extrait de la Zeitungshalle], C. G. Vogler, Bruxelles, 1847-48.

64. Betrachtungen über den Zustand der Polen (Réflexions sur l'état de la Pologne), Bruxelles, 1848 ; Krysiński A. L., La question Polonaise dans l'état actuel de l'Europe, par ... ancien secrétaire de l'état, aide de camp du général en chef conseiller d'état, Meline cans et Comp., Bruxelles, 1848.

65. Terlecki H. Z., Relation de l'emprisonnement d'un prêtre catholique à Dresde, pendant les événements du mois de mai 1849, J. B. de Mortier, Bruxelles, 1849.

66. Lettre de Lubliner L. à Schmitt H., 25/12/1859, Bruxelles, Ossolineum (Wrocław), $\mathrm{n}^{\circ}$ 5916, pp. 453-456. Au sujet des contacts entre Lelewel et son pays d'origine (ses études historiques furent p. ex. publiées en 18 volumes en 1858 à Poznań), voir Kalembka Sł., Liens de Lelewel avec la Pologne durant son séjour à Bruxelles, actes du colloque «Joachim Lelewel à Bruxelles de 1833 à 1861 », op. cit. pp. 103-111.

67. [Czacki, Włodzimierz], Rome et la Pologne, avec documents ecclésiastiques officiels, A. Mertens et fils, Bruxelles, 1864 ; Lubliner L., La Pologne devant le Sénat francais [Extrait du journal Le Levant, Bruxelles, 31 décembre 1863], Ch. et A. Vanderauwer, Bruxelles, 1863; Sawaszkiewicz L. L., La politique anglo-polonaise et la diplomatie générale, Bruxelles, 1864 ; Tyszkiewicz T., Notes pour servir à l'intelligence des affaires de Pologne, N.1. $1^{\text {er }}$ Janvier 1864. Les Czartoryski, Leys et Seeraert, Bruxelles, 1864.

68. Segur D'Aguesseau (de) R., Mowa hrabiego ... w interesie Polski, miana $w$ senacie francuskim 17go grudnia 1863 roku. Ttumaczenie Józefa Wiena (Discours du comte ... dans l'intérêt de la Pologne, donné au sénat français le 17 décembre 1863. Traduction : Józef Wien), J. Nys, Bruxelles, 1863 ; La Pologne devant le tribunal du congrès européen. Publié simultanément en polonais, S. Gerstmann, Bruxelles, 1864 ; Sprawa polska przed trybunałem kongresu europejskiego. Głos z kraju (La cause polonais devant le tribunal du congrès européen. Voix du pays), J. Nys, Bruxelles, 1864 ; Swierszcz B., Pokój w Villa-Franca, jego wpływ na sprawy europejskie (La Paix de Villa-France, son influence sur les affaires européennes), S. Gerstmann, Bruxelles-Leipzig, 1864.

69. Bukaty T., Lettres sur l'opuscule de P.J.Proudhon: si les traités de 1815 ont cessé d'exister?, Bruxelles, 1864 ; Czyński J., Monsieur Proudhon, op. cit., ; Wicherski F., Lettre sur l'opuscule de P. Proudhon, Bruxelles, 1864.

70. Tyszkiewicz T., Ecrits sur la Pologne (1862-1864), Bruxelles, 1865.

71. Ostrowski J. B., La France, la Pologne et le Prince Napoléon Bonaparte, Ch. Muquardt, Bruxelles, 1868 ; Swierszcz B., Napoleon III et les compensations territoriales de la France, traduit du polonais par Edmond Callier, Bruxelles-Poznań, 1868. 
72. Seroczyński [=Syroczyński] L., Études sur la Russie contemporaine, le nihilisme, Ed. Coll., Bruxelles, 1867 ; Syroczyński L., Le Panslavisme [Extr. de la Revue de Belgique, 15 Juillet 1869], Bruxelles, 1869.

73. Ronin Vl., «Le publicisme russe en Belgique au milieu du XIX ${ }^{\mathrm{e}}$ siècle », in : Revue des Pays de l'Est, vol. XXXII, nn 1-2, 1991, pp. 2-24.

74. Borejsza J. W., « Prasa Zjednoczenia Emigracji Polskiej (1866-1870)» (La presse de l'Union de l'Émigration polonaise [1866-1870]), in : Rocznik Historii Czasopiśmienictwa Polskiego, vol. III, 1964, p. 90.

75. Par ex. : Lettre de Heltman W. à la rédaction de la revue Strzech (28/12/1871, Bruxelles), Ossolineum (Wrocław), nº 12 183, p. 19.

76. Peut-être bien grâce à l'entremise de W. Wojcicki, le rédacteur du Kłosy. Voir : Lettre de Jeż T. T. à Wojcicki K. W. (18/06/1868, Bruxelles), Bibliothèque Jagellone de Cracovie, nº 7832, pp. 170-171.

77. Jeż T. T.., «Belgija i Belgowie. Studyjum » (La Belgique et les Belges. Etude), in : Niwa, vol. I et II, 1872 ; Nowy Korbut, op. cit. p. 390.

78. Van Heuckelom Kr., "Listy z Belgii van Włodzimierz Wolski en Henryk Merzbach. Het laatnegentiende-eeuwse België in de ogen van twee Poolse emigranten » (Les lettres de Belgique de Włodzimierz Wolski et de Henryk Merzbach. La Belgiqie de la fin du XIx ${ }^{\mathrm{e}}$ siècle dans les yeux de deux émigrés polonais), in : Oost-Europa Tijdingen. Bijdragen tot de studie van Centraal- en OostEuropa, vol. XXIII/XL, n 2, 2001, pp. 48-57.

79. Qui en 1867 écrivait par exemple pour le Kłosy, fût-ce à partir de Dresde; voir : Lettre de Skiba W. [Sabowski W.] à Wojcicki K. Wł. (02/06/1867, Dresde), Bibliothèque Jagellone de Cracovie, $n^{\circ} 7833$, p. 63. Władysław Sabowski retourna en 1869 en Galice, où il devint un célèbre journaliste. Il n'est pas cité dans le Nowy Korbut.

80. C'est ainsi que j'ai trouvé une courte lettre écrite à Liège dans laquelle Romuald Majer remercie Józef Ignacy Kraszewski pour les livres envoyés : Lettre de Majer R. à Kraszewski J. I. (06/02/1869, Liège), Bibliothèque Jagellone de Cracovie, $\mathrm{n}^{\circ} 6518$, p. 292. Voir également: Zarzycka M., art. cit. pp. 83 \& 91.

81. Kamiński K., « Materiały do życia i twórczości Włodzimierza Wolskiego » (Matériel sur la vie et l'oeuvre de Włodzimierz Wolski), in : PamiĘtnik Literacki, vol. LXV, nº 1, 1974, pp. 143-169 et Nowy Korbut, op.cit. (pour Merzbach: vol.8, pp.374-375). De toute évidence, tous deux s'exerçaient également à d'autres genres, et Merzbach écrivit p. ex. des commentaires dans les Nowiny Warszawskie au sujet de l'achat d'une peinture de Copernic pour un musée spécialisé (voir Lettre de Merzbach $\mathrm{H}$. à Kraszewski J. I. (09/04/1879, Bruxelles), Bibliothèque Jagellone de Cracovie, $n^{\circ} 6519$, p. 439a [=539]) ou des critiques litéraires dans le Dziennik Poznański (BJ, 4818 [voir lettre de id. à id. (23/03/1866, Bruxelles) ; une critique de l'ouvrage Tytan-Arion z Koryntu par Alkar).

82. Lettre de Merzbach H. à Kraszewski J. I. (28/07/1879, Bruxelles), Bibliothèque Jagellone de Cracovie, $\mathrm{n}^{\circ} 6519$, p. 444a [=544].

83. Lettre de Merzbach H. aux établissements Gebethner et Wolff à Varsovie (27/08/1889, Spa), Bibliothèque nationale de Varsovie, $n^{\circ}$ 7292, pp. 10-11. 
INDEX

Index géographique : Belgique, Bruxelles, Pologne

Index chronologique : romantisme, XIXe siècle

Mots-clés : édition, émigration, émigration polonaise, exil, histoire de la Pologne, littérature

polonaise, presse, romantisme polonais 